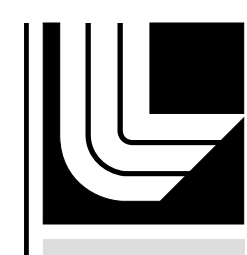

LAWRENCE LIVERMORE N A T IO N A L LABORATORY

Near infrared spectral imaging of explosives using a tunable laser source

G.L. Klunder, E. Margalith, L.K. Nguyen

April 12, 2010

SPIE Defense, Security, and Sensing

Orlando, FL, United States

April 5, 2010 through April 9, 2010 
This document was prepared as an account of work sponsored by an agency of the United States government. Neither the United States government nor Lawrence Livermore National Security, LLC, nor any of their employees makes any warranty, expressed or implied, or assumes any legal liability or responsibility for the accuracy, completeness, or usefulness of any information, apparatus, product, or process disclosed, or represents that its use would not infringe privately owned rights. Reference herein to any specific commercial product, process, or service by trade name, trademark, manufacturer, or otherwise does not necessarily constitute or imply its endorsement, recommendation, or favoring by the United States government or Lawrence Livermore National Security, LLC. The views and opinions of authors expressed herein do not necessarily state or reflect those of the United States government or Lawrence Livermore National Security, LLC, and shall not be used for advertising or product endorsement purposes. 


\title{
Near infrared spectral imaging of explosives using a tunable laser source
}

\author{
Eli Margalith ${ }^{*},{ }^{1}$, Lam K. Nguyen ${ }^{1}$ and Greg Klunder ${ }^{2}$ \\ OPOTEK, Inc., 2233 Faraday Ave., Suite E, Carlsbad, CA USA 92008
}

\begin{abstract}
Diffuse reflectance near infrared hyperspectral imaging is an important analytical tool for a wide variety of industries, including agriculture ${ }^{1,2}$, consumer products, chemical $^{3}$ and pharmaceutical development and production ${ }^{4,5}$. Using this technique as a method for the standoff detection of explosive particles is presented and discussed. The detection of the particles is based on the diffuse reflectance of light from the particle in the near infrared wavelength range where $\mathrm{CH}$, $\mathrm{NH}, \mathrm{OH}$ vibrational overtones and combination bands are prominent.

The imaging system is a NIR focal plane array camera with a tunable OPO/ laser system as the illumination source. The OPO is programmed to scan over a wide spectral range in the NIR and the camera is synchronized to record the light reflected from the target for each wavelength. The spectral resolution of this system is significantly higher than that of hyperspectral systems that incorporate filters or dispersive elements. The data acquisition is very fast and the entire hyperspectral cube can be collected in seconds. A comparison of data collected with the OPO system to data obtained with a broadband light source with LCTF filters is presented.
\end{abstract}

Key Words:

Hyperspectral Imaging; Chemical Imaging; Trace-Analysis; Standoff Detection

\section{INTRODUCTION}

Increased security measures due to the continuous threat of terrorist activities have placed strong demands on the development of new technologies to detect explosive materials. In addition to airport screening, seaports across the world are increasing their screening of cargo containers for suspicious cargo, and first responders are in constant need of improved technologies may come upon a suspect package or powder. ${ }^{6}$ A number of screening technologies are available for detecting explosives. ${ }^{7}$ The most prevalent method used at airports is the ion mobility spectrometer (IMS). This method works by swiping the baggage with a filter or some type of collection membrane and thermally desorbing the residue in the IMS instrument. Although this technique is fast and relatively reliable, its inherent weakness is that it requires that a particle be collected on the swipe. In this study we describe a system capable of acquiring images of an area and highlight particles or materials that could be suspect materials based on spectral signatures. The initial scope of this study was to demonstrate the performance potential of this technique for advanced screening with the possibility of identification of materials based comparisons to a predetermined library. The primary objectives of the current effort is to demonstrate that NIR imaging is capable of highlighting explosive materials and determining approximately what the limits of detection are for minimum particle size.

Near infrared (NIR) spectroscopy measures the absorption of light by molecules in the wavelength range from 700-2500 $\mathrm{nm}$. The predominant absorption features are due to the $\mathrm{CH}, \mathrm{OH}, \mathrm{NH}$ vibrational overtones and combination bands. Since many energetic materials contain these groups, they will have unique spectra in the NIR and thus, it should capable of identifying many explosives.

\footnotetext{
*elimar@opotek.com

1 OPOTEK Inc.

${ }^{2}$ Forensic Science Center, Lawrence Livermore National Laboratory, Livermore, CA 94551.
} 
We present and compare performance of two HyperSpectral instruments:

- A conventional NIR imaging instrument which utilizes a broad band light source to illuminate the samples and a Liquid Crystal Tunable Filter (LCTF) to select the wavelength band recorded by the NIR camera.

- A system that utilizes a tunable OPO laser to illuminate the target with a very narrow wavelength light source that is tunable over a wide range of wavelengths without the need for an additional filter.

Both systems operate in a stare down mode, where the image of a stationary sample is acquired in consecutive frames, each at a different wavelength. The same NIR camera is used by both systems to record the Hyperspectral cube.

\section{MATERIALS AND METHODS}

Spectra were acquired with two systems; one incorporates a broadband illumination with tunable filters, and the other utilizes a tunable laser (OPO) to illuminate the target.

\subsection{Sample Preparation}

Samples were analyzed by placing the powders or crystals on to a cellulose based swipe material with no additional preparation required. Dimethyl dinitrobutane (DMNB), diphenylamine (DPA) and sugar were from Sigma Aldrich. The explosive materials, 2,4,6-trinitrotoluene (TNT), 1,3,5-trinitro-1,3,5-triazacyclohexane (RDX), and pentaerythritol tetranitrate (PETN) were acquired from XM Materials as non-explosive training aids for canines at $8 \%$ (TNT, RDX) and $4 \%$ (PETN) by weight on silica.

\subsection{NIR reference spectra}

The reference spectra were acquired using an Analytical Spectral Devices Inc. Labspec Pro NIR spectrometer which has a tungsten halogen light source and 3 detectors to cover the spectral range from $350-2500 \mathrm{~nm}$. Fiber optic bundles were used to transmit the light to and measure the reflected light from the sample. Spectra were scanned in $1 \mathrm{~nm}$ steps with a spectral resolution of approximately $10 \mathrm{~nm}$.

\subsection{Imaging with broadband illumination and LCTF}

The NIR imaging system assembled at Lawrence Livermore National Laboratories (LLNL) consists of a broadband light source to illuminate the target (scene) where Liquid Crystal Tunable Filters (LCTF) are placed between the camera and the target to select the wavelength band that was collected by the NIR camera (Error! Reference source not found.Figure 1.).

The NIR camera is a temperature stabilized InGaAs focal plane array (AlphaNIR, Indigo Systems Inc., Goleta, Ca) of 320 x 256 elements that is sensitive to light from $400-1700 \mathrm{~nm}$. The liquid crystal tunable filter (MIR-20, CRI Inc. Woburn, Ma.) $20 \mathrm{~nm}$ bandwidth, 50\% transmission at each wavelength setting and is tunable from $900-1800$ $\mathrm{nm}$. An integrated software package (IRVista, Indigo Systems Inc.) is



Figure 1 NIR Hyperspectral camera with tunable filter. used to control both the camera and the tunable filter. The LCTF was scanned from 1000 to $1650 \mathrm{~nm}$ with integration times were of $32 \mathrm{~ms}$ at each step, however, chromatic aberrations of the lens limited the usable scan range to several hundred $\mathrm{nm}$. Non-uniformity correction (NUC) and bad pixel replacement (BPR) were performed to calibrate the camera based on the manufacturer guidelines. A 5" x 5" diffuse reflectance standard that has greater than $98 \%$ reflectivity over the entire range of operation 1000-1700 nm (LabSphere, model \# 
SRT-99-050) was illuminated with a tungsten halogen light source. When imaging, samples were placed on a white paper background and the camera system set on an adjustable height tripod.

\subsection{Tunable Laser System}

The second system is a commercial product (HySPECTM, OPOTEK, Carlsbad, CA) which incorporates an OPO as the illumination source. The system head, presented in Figure 2, contains the same camera as in the LLNL system, and a fiber bundle that delivers the OPO beam onto the target. The lens used in the present configuration is a $50 \mathrm{~mm}, f / 1.4$, chromatically corrected between $0.9 \mu \mathrm{m}$ up to $1.7 \mu \mathrm{m}$ (Electrophysic). The system schematic is presented in Error! Reference source not found.Figure 2 .

- The OPO based instrument offers significant advantages including:

- High spectral resolution; better than $1.5 \mathrm{~nm}$.

- The OPO generates very short pulses, on the order of $5 \mathrm{~ns}$, resulting in very high instantaneous power (for high quality images) and very low average power (no heating of the sample).

- The short pulse enables operation in ambient light.

- The high instantaneous power enables analysis of large as well as small samples. From a few $\mathrm{mm}$ to $10 \mathrm{~s}$ of $\mathrm{cm}$.

- Fast scan-rates, the entire hyperspectral cube can be collected in a few seconds

- Efficient coupling to optical fibers, enabling flexible delivery of the light to the sample.

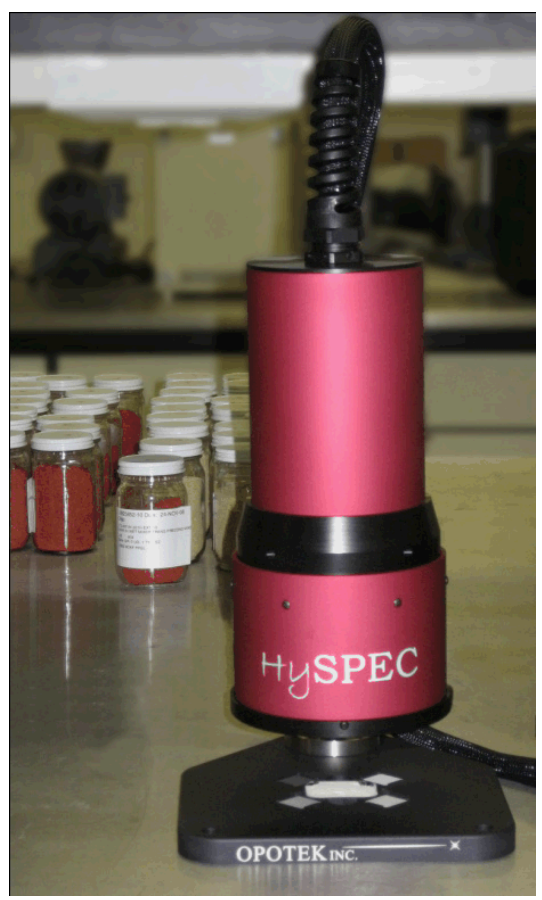

- Actual wavelengths are recorded and the reflectance signal is calibrated and corrected for linearity at each wavelength in real-time.

Figure 2 HySPEC System

\subsection{Control Software}

The control software consists of two main layers: a userinterface layer that provides high-level control of the main functions and a hardware control layer that provides low-level interfacing with the hardware sub-systems. To perform a scan, the user simply specifies the starting wavelength, the ending wavelength, and the desired spectral resolution. The low-level layer of the software takes over and controls all the functions of the illumination source and the camera. The laser is switched on and off automatically; the wavelength is changed as specified in the scan parameters, and the camera is synchronized to the laser's pulses for recording the images.

\subsection{Non-Uniformity Calibration}

The illumination as seen by the camera is almost never uniform. This is due to the combination of non-uniformity in illumination and distortion by the lens. Furthermore, each pixel of the FPA has different sensitivities compared to its

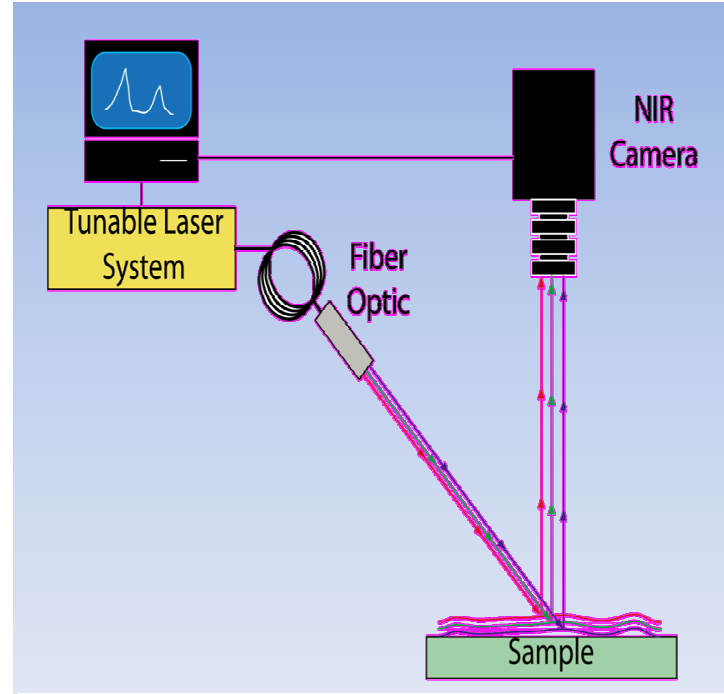

Figure 3 Schematic presentation of the HySPEC neighbors. The recorded data has to be independent of the location of the sample in the camera's FOV. Therefore, spatial non-uniformities of the illumination, the lens and the camera have to be corrected. The correction technique is straight-forward. A plate with a surface having uniform and calibrated reflectivity is placed on the target area such that it 
fills the entire FOV of the camera. The camera is then directed by the control software to collect a series of "dark" frames followed by a series of frames taken while the plate is illuminated by the laser light. The "dark noise" and the illumination at each pixel are recorded and stored to serve as a calibration matrix. Since the reflectivity of the plate is uniform across the entire FOV the recorded data at each pixel factors in the non-uniformity of the illumination, the lens, and the FPA. The calibration matrix is valid for a given lens and illumination geometry.

The shape of illumination and the spectral response of the camera change with the illumination wavelength. Therefore, the calibration is performed at regular spectral intervals across the spectral range of the system. The calibration points are between $20 \mathrm{~nm}$ to $50 \mathrm{~nm}$ apart. Correction factors between calibration points are interpolated.

\subsection{Non-Linearity Correction}

A unique feature of the system is that the reflectivity of the target is presented in absolute values, which are system independent. This is achieved by correcting the recorded signals for variations in the illumination intensity and nonlinearity of the camera's response.

These corrections are done in real time for each recorded frame using the reflected signal collected from four calibration disks that are embedded within the FOV of the camera. The software assigns the known absolute reflectivity of each disk to the signal value (counts) averaged over the disk surface. The software then uses these values to generate a calibration curve to correct for fluctuations in the illumination intensity as well as for the non-linearity of the camera.

\subsection{Data Collection}

The system has three scan modes: Standard-Scan and Fast-Scan. Standard-Scan is designed to obtain the best signal-tonoise ratio using multiple frame averaging. This scan is typically used to record the spectra of pure material which is used as reference (library). Fast-Scan is optimized for speed with 20 images recorded per second, each at a different wavelength

In Standard-Scan mode, the operator specifies the starting and ending wavelengths within the range, the step size, or spectral resolution (in $\mathrm{nm}$ ), and the number of frames to be recorded at each wavelength.

In Fast-Scan, the operator specifies the starting and ending wavelengths and the approximate step size (spectral resolution) as in the Standard-Scan. When the scan is initiated the laser is tuned to the starting wavelength and then it tuned continuously towards the ending wavelength. The laser fires continuously during the scan at 20 pulses per second and the camera records every pulse. During the scan, a spectrometer (USB4000, Ocean Optics, Dunedin, FL) records the actual wavelength of each pulse in real time.

The number of recorded frames is approximately equal to the scanned wavelength range divided by the desired step size, and the duration of the scan is the number of frames divided by 20 (the pulse repetition rate of the laser). e.g. a scan from $1000 \mathrm{~nm}$ to $1700 \mathrm{~nm}$ with a $2 \mathrm{~nm}$ resolution will generate 350 data points (frames) and will be completed in 17.5 seconds.

\section{RESULTS AND DISCUSSION}

\subsection{Data Obtained with the LLNL System}

Diffuse reflectance spectra for three different common explosive materials, TNT, PETN, and RDX, are presented in Figure 4Error! Reference source not found.. The spectra were acquired with the NIR spectrometer and the data are presented limited to the wavelength range achievable with the NIR camera system. A library of these spectra was developed and principal component analysis was used to identify unknown materials. Each spectrum is unique and there are common peaks at approximately $1650 \mathrm{~nm}$ due to $\mathrm{C}-\mathrm{H}$ vibrational overtones that appear in the explosive spectra but not in the spectra of other materials studied. Although, other materials could have absorption in this range, $1650 \mathrm{~nm}$ could be considered as one of the primary discriminating wavelengths when using NIR imaging as a screening technique. 


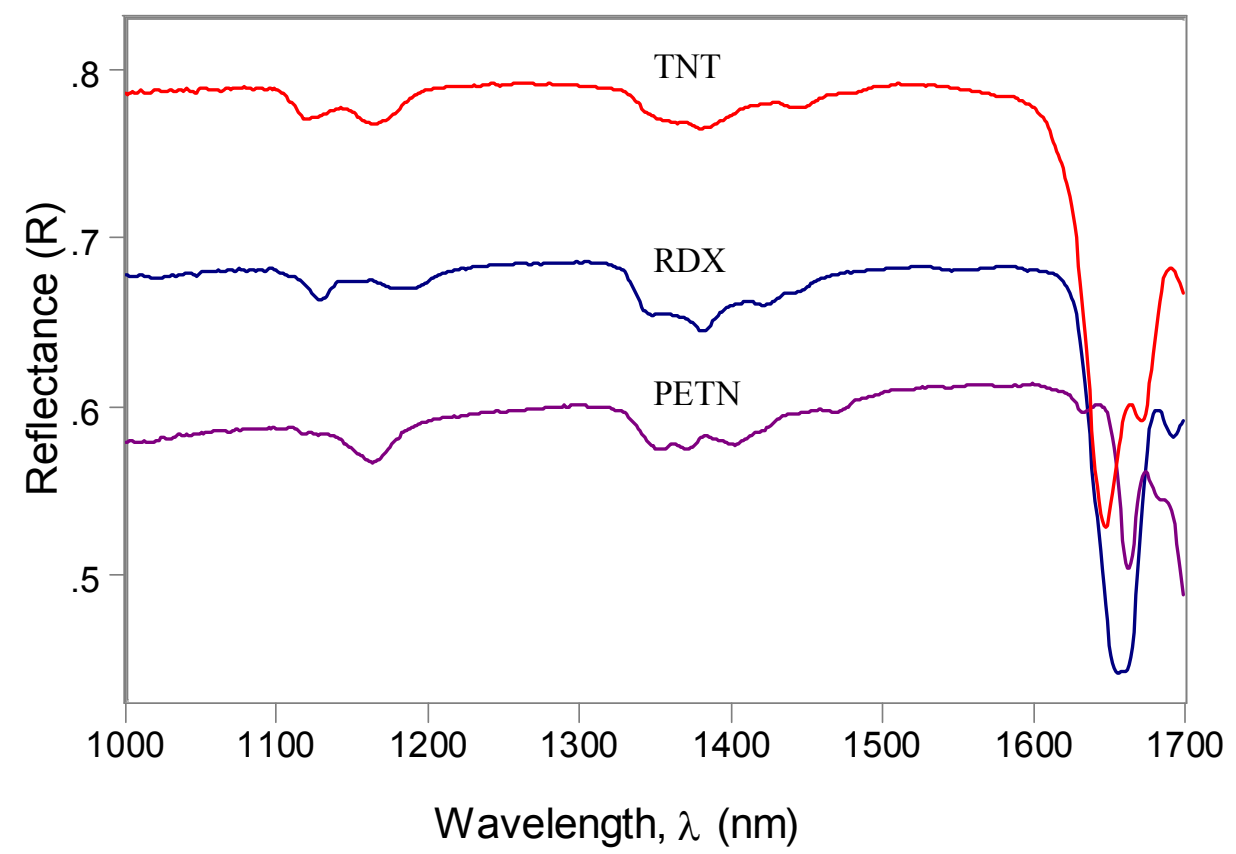

Figure 4 Spectra of TNT, RDX and PETN (spectral resolution $\sim 10 \mathrm{~nm}$ ).

Figure 5 shows 3 NIR images of a piece of TNT on a cardboard box taken from a standoff distance of approximately 6 feet. In Figure 5A, the image at $1400 \mathrm{~nm}$ was referenced against $1100 \mathrm{~nm}$ and the TNT particle is not visible since there is no significant absorption at either wavelength. Figure 5B and 5C, use an absorption band of TNT at $1650 \mathrm{~nm}$ referenced against $1600 \mathrm{~nm}$ or $1100 \mathrm{~nm}$, respectively, that clearly highlight the particle. One of the goals of these tests was to estimate minimum detectable particle size. Many factors including field of view, distance from the detector, light source, background reflectivity, and discrimination from other particles need to be considered in determining a LOD. TNT was selected as a representative explosive for the LOD study and sugar was included as a neutral material to discriminate against.
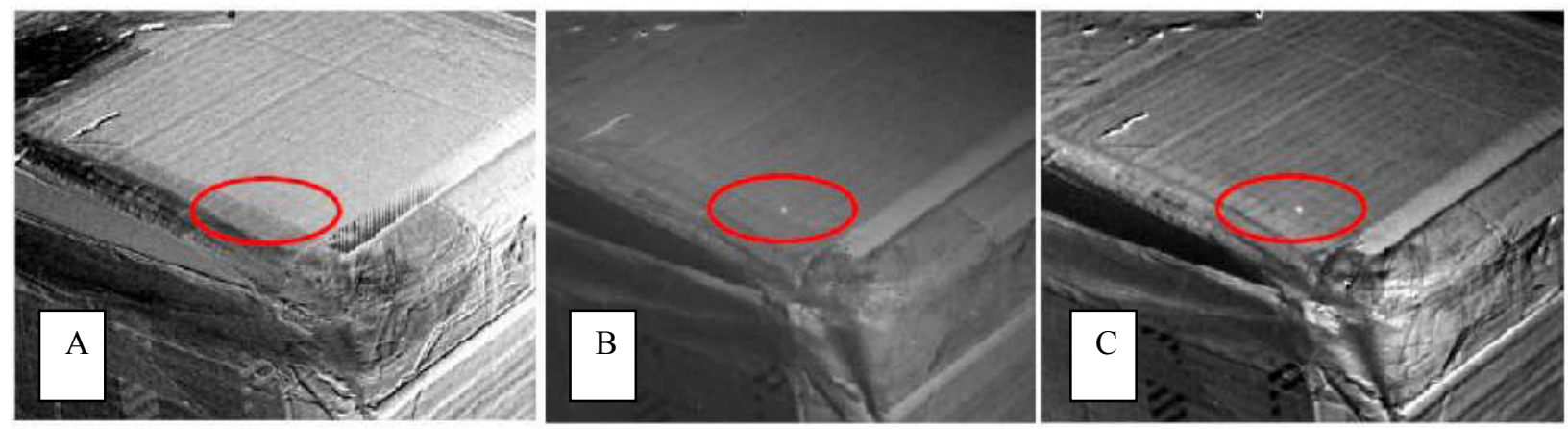

Figure 5 Particle of TNT on a cardboard box

Figure 6 compares the diffuse reflectance spectra of these two materials. Both materials have relatively strong absorption bands at $1650 \mathrm{~nm}$, however, TNT could easily be discerned from the sugar using a relatively short wavelength scan. With a field of view (FOV) of 5" x 6" and a standoff distance of 3', particles of TNT could be spectrally discerned from the sugar. The particles were between $200 \mu \mathrm{m}$ and $1 \mathrm{~mm}$ diameter, which is near the spatial resolution for the $256 \mathrm{x} 320$ focal plane array camera. These results were repeated with a telephoto lens at a distance of 10', however, the light source was kept at a distance of 3' and the intensity increased. With the larger lens, the spectral range was limited to $100 \mathrm{~nm}$ due to more significant chromatic aberrations. 


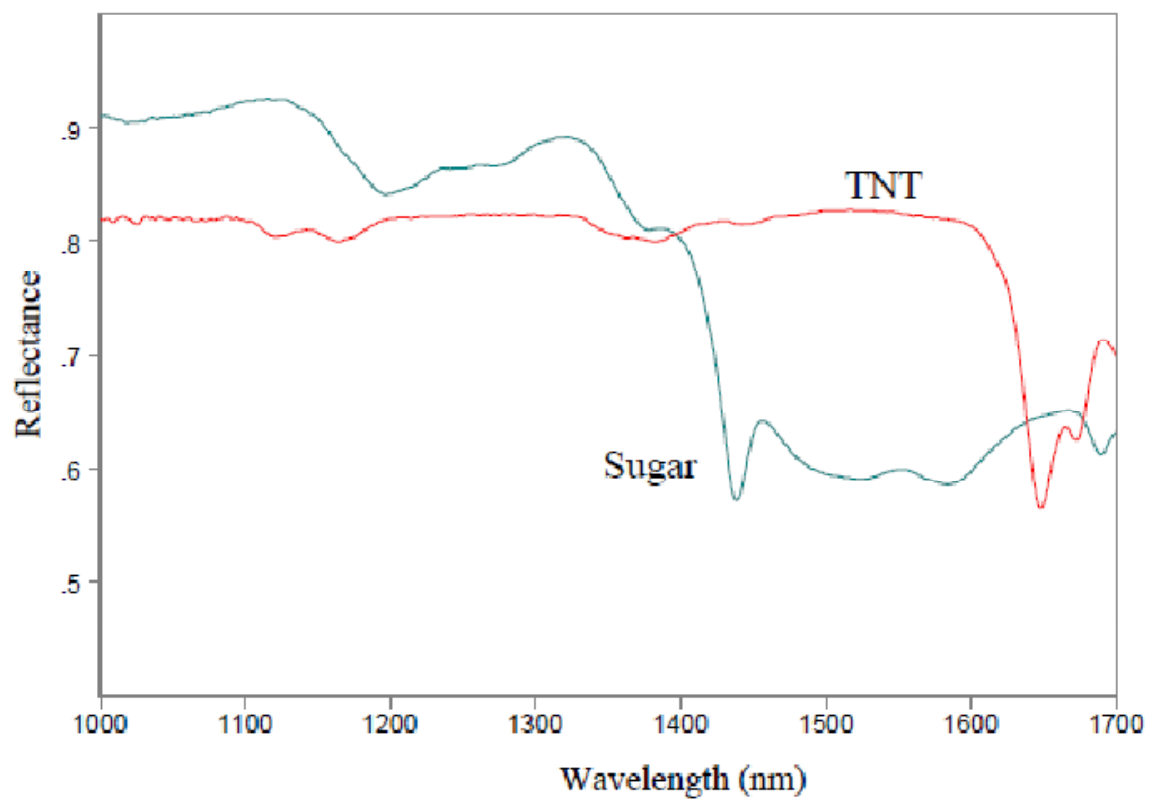

Figure 6 Spectra of Sugar and TNT (spectral resolution $\sim 10 \mathrm{~nm}$ )

\subsection{Data Obtained with the HySPEC}

A second objective of the tests was to investigate the detection of explosives and explosive related materials on swipe. These tests were conducted with the HySPEC, taking the advantage of the high spectral resolution and the fast acquisition time. The spectra of a swipe material, a taggant material (2,3 Dimethyl 2,3 Dinitrobutane, DMNB), a stabilizer (diphenylamine, DPA) are presented in Figure 7Error! Reference source not found.. The spectra were recorded at $1 \mathrm{~nm}$ resolution, which is significantly higher than the spectra collected with the previous system.

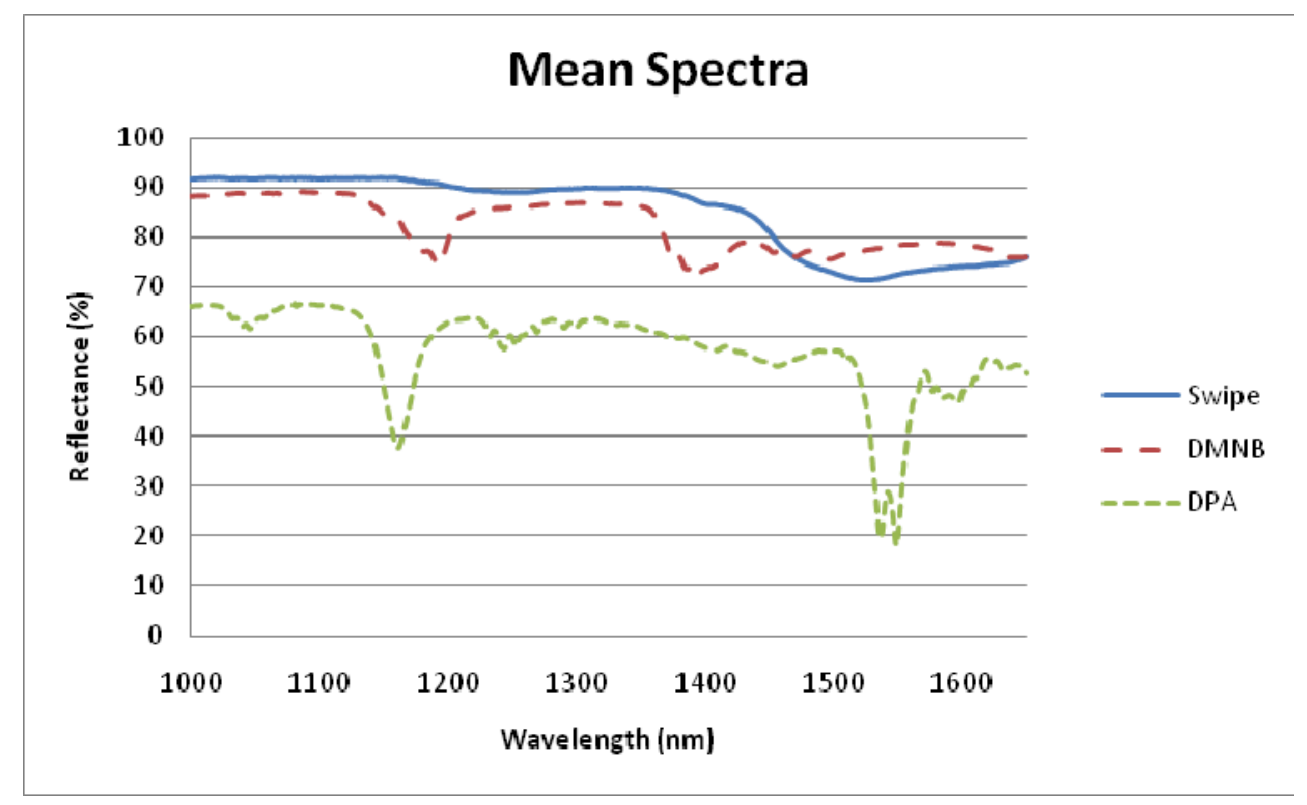

Figure 7 Spectra of Swipe, DMNB, and DPA 
The first test was to try to detect DPA by itself. A crystal of DPA was crushed with the tip of a toothpick. The small particles of DPA that were stuck to the toothpick were deposited on to a thin strip, $5 \mathrm{~mm}$ wide, of the swipe material by wiping the toothpick on the swipe. Principle Component Analysis (PCA) was used to locate the particles of DPA. These particles show up as PC2 capturing $2.66 \%$ of the variance in Figure 9.

The second test was to try to detect DMNB by itself. Using similar technique as describe above, a small amount of DMNB was deposited on to a thin strip of the swipe. The results of the PCA are shown below. The DMNB showed up as PC3 capturing 1.37 of the variance, as presented in Figure 8.



Figure 8 DPA on Swipe

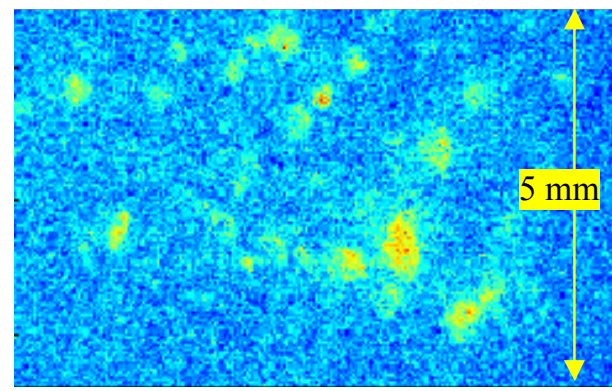

Figure 9 DMNB on Swipe

The next set of tests was directed to detection of explosives. The spectra of pure TNT, RDX, and PETN are presented in Figure 10.

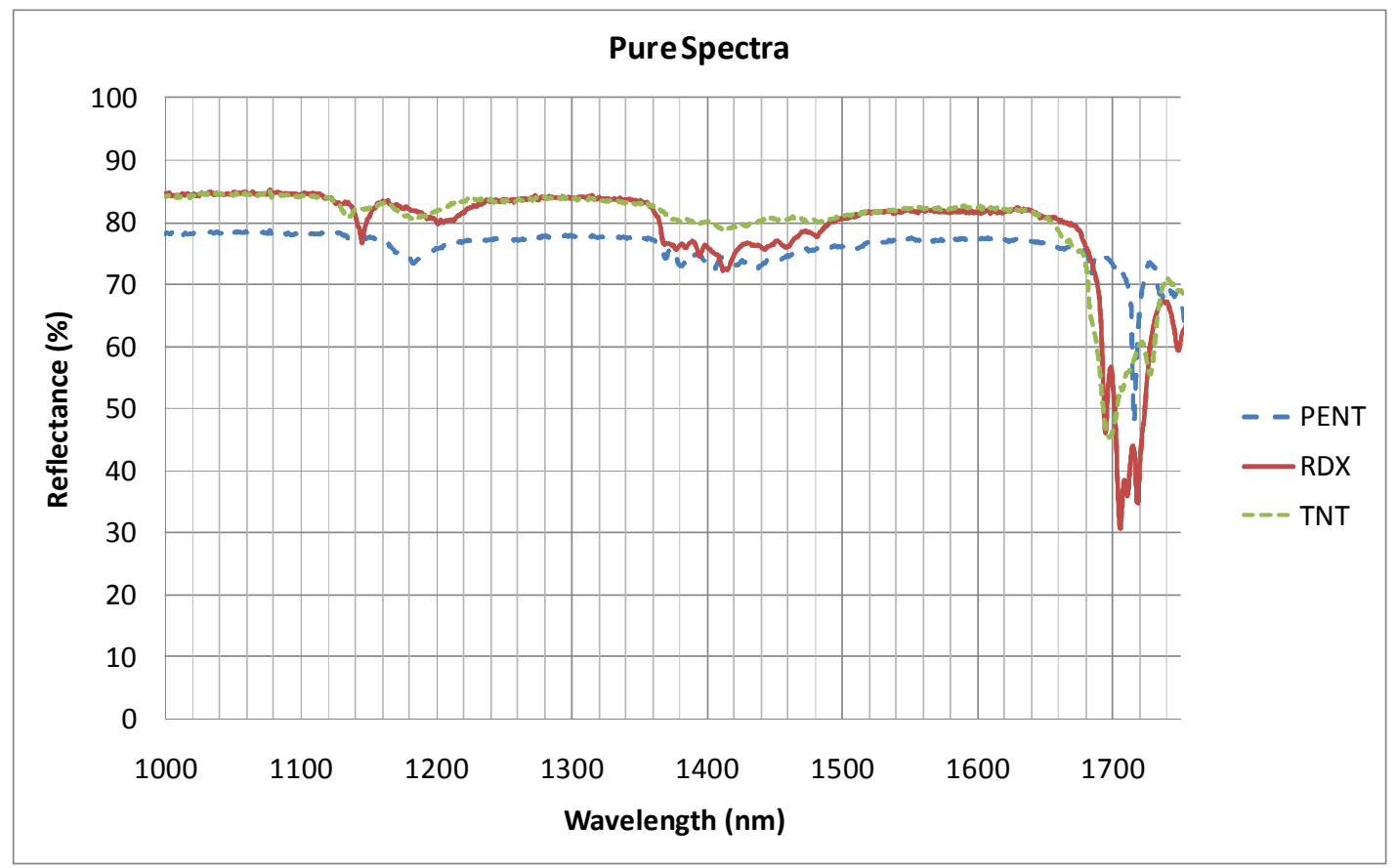

Figure 10 Spectra of PETN, RDX, and TNT (spectral resolution $1 \mathrm{~nm}$ ) 
As can be seen in the spectra, these materials have relatively sharp peaks around $1700 \mathrm{~nm}$. The high resolution of the HySPEC system provides more detailed spectra compared to the data presented in Figure 4. The improved spectral resolution will provide a better ability to discriminate against other materials and should lead to improved limits of detection.

Small particles of these explosives were placed on swipe. The weight of each material was measured with analytical balance having a minimum measurement level and resolution of $0.1 \mathrm{mg}$. Spectral images were taken with $1 \mathrm{~nm}$ resolution and analyzed with PCA.

Figure 11 is a false color presentation of TNT on swipe material. The weight of the TNT was approximately $0.5 \mathrm{mg}$. From the analysis, $>90$ pixels have significant presence of TNT. The pixel size in the camera's array is $30 \mu \mathrm{m}$. The maginification of the camera's lens is $\mathrm{x} 4$, therefore, the pixels size on the target is $120 \mu \mathrm{m}$. The area of 90 pixels is about $1.3 \mathrm{~mm}^{2}$.

Figure 12 presents a swipe with traces of RDX. The weight of the RDX was below the resolution of the scale, or less than $0.1 \mathrm{mg}$. The number of pixels with RDX presence is more than 70. Figure 13 presents a swipe with traces of PETN. The weight of the RDX was below the resolution of the scale, or less than $0.1 \mathrm{mg}$. The number of pixels with PETN presence is more than 50 .

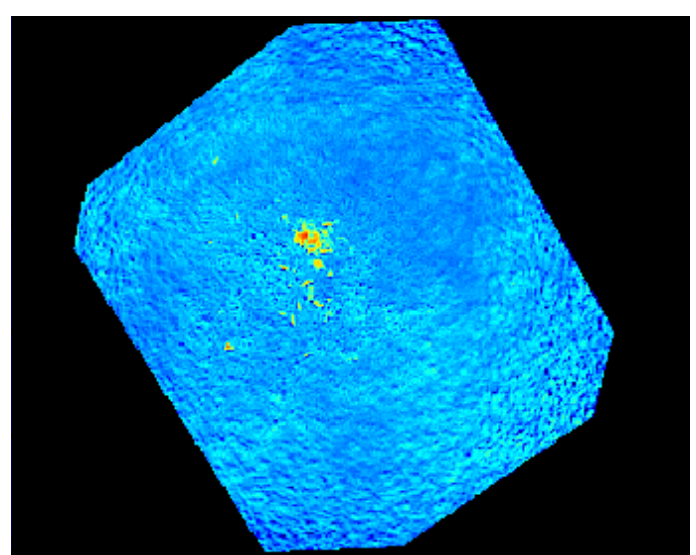

Figure 11 TNT on swipe

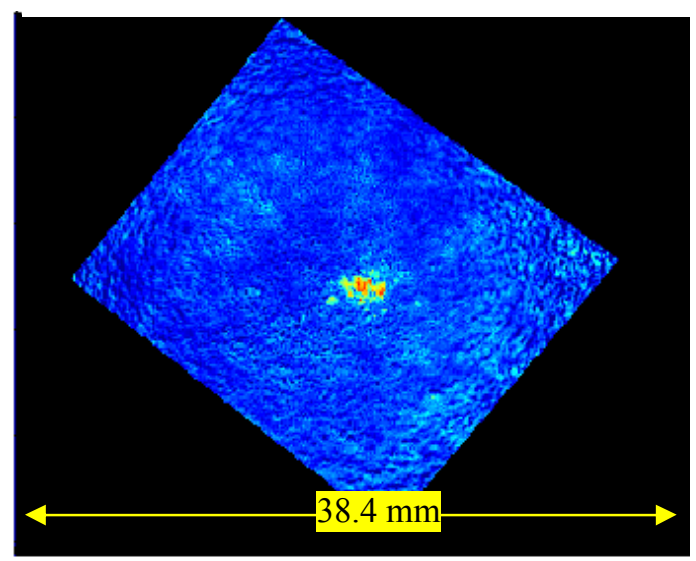

Figure 13 PETN on Swipe

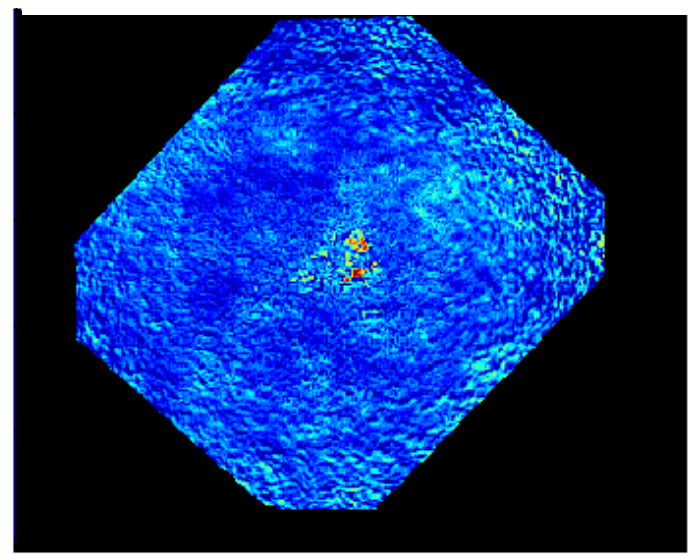

Figure 12 RDX on Swipe 


\section{CONCLUSIONS}

We have demonstrated that near infrared imaging can be a valuable tool for screening and potentially identifying explosive particles. The current limits of detection demonstrated are rather high but we have not reached yet the detection limits. There is a direct correlation between the LOD and the time it takes to complete the tests. Future studies are planned to investigate the LOD for different explosives as while minimizing the acquisition time.

Improvements in hardware will also be considered. InGaAs FPA cameras have already improved to $640 \mathrm{x} 512$ elements and are expected to continue to improve. Extending the wavelength range to $2400 \mathrm{~nm}$ will enable better spectral discrimination from background or more positive identification. Focal plane arrays made with $\mathrm{HgCdTe}$ (MCT) detector provide excellent response out to these longer wavelengths will be tested.

In addition to the experimental improvements, there are a number of fundamental studies that should be investigated to determine the ultimate limitations of NIR imaging spectroscopy. A broader more fundamental determination of detection limits that relates the minimum particle size (diameter and thickness) and the absorption and scattering properties of the material could be determined. One of the key tests of performance will be to determine how well explosive particles can be discriminated from common background materials. Further evaluations should explore how well explosives can be detected on different materials, e.g. clothes, packaging material, soil, and vegetation. Ideally an explosive detection/screening system will be able to respond in real time. The optical configuration, number of wavelengths interrogated, and signal processing algorithm needed to provide a satisfactory response would have to be redesigned to provide real time information.

Lawrence Livermore National Laboratory is operated by Lawrence Livermore National Security, LLC, for the U.S. Department of Energy, National Nuclear Security Administration under Contract DE-AC52-07NA27344.

\section{REFERENCES:}

[1] Kim, M. S., Chen, Y. R. and Mehl, P. M., "Hypersperspectral Reflectance and Fluorescence Imaging System For Food Quality and Safety", American Society of Agricultural Engineers 44(3), 721-729 (2001).

[2] Kim, M. S., Lefcourt, A. M., Chao, K., Chen, Y. R., Kim, I. and Chan, D. E., "Multispectral Detection of Fecal Contamination on Apples Based on Hyperspectral Imagery: Part I: Application of Visible and Near-Infrared Reflectance Imaging", Transactions of the ASAE 45(6), 2027-2037 (2002).

[3] Dowrey, A. E., Story, G. M., and Marcott, C., [Spectrochemical Analysis Using Infrared Multichannel Detectors], Blackwell Publishing, Oxford, UK, 175-188 (2005).

[4] Koehler, F. W., Lee, E., Kidder, L. H. and Lewis, E. N., "Near Infrared Spectroscopy: the Practical Chemical Imaging Solution", Spectroscopy Europe 14(3), 12-19 (2002).

[5] Lewis, E. N., "A Near Infrared View of Pharmaceutical Formulation Analysis", NIR News 12(3), 16-18 (2001).

[6] Steinfeld, J.I.; Wormhoudt, J. Annu. Rev. Phys. Chem. 1998, 49, 203-232. "Explosive Detection: A challenge for physical chemistry".

[7] Yinon, J.; Zitrin, S. Modern Methods and Applications in Analysis of Explosives, J. Wiley and Sons, 1993, New York, ISBN 0471938947. 
[8] Burns, D.A.; Ciurczak, E.W. Handbook of Near-infrared (NIR) Analysis, $3^{\text {rd }}$ Ed., CRC Press, 2007, ISBN 978-0-84937393-0. 